Critique de l'expérimentation: paradigmes politiques et technologies gouvernementales

\title{
Le consensus expérimentaliste et ses limites
}

\section{Marc Maesschalck}

\section{OpenEdition}

\section{Journals}

Édition électronique

URL : https://journals.openedition.org/grm/3250

DOI : $10.4000 / \mathrm{grm} .3250$

ISSN : $1775-3902$

Éditeur

Groupe de Recherches Matérialistes

Référence électronique

Marc Maesschalck, "Le consensus expérimentaliste et ses limites », Cahiers du GRM [En ligne], 18 | 2021, mis en ligne le 28 septembre 2021, consulté le 01 octobre 2021. URL : http:// journals.openedition.org/grm/3250; DOI : https://doi.org/10.4000/grm.3250

Ce document a été généré automatiquement le 1 octobre 2021.

(c) GRM - Association 


\title{
Le consensus expérimentaliste et ses limites
}

\author{
Marc Maesschalck
}

1 Parmi les innovations apportées par le tournant pragmatique des sciences sociales qu'on peut situer à la fin des années 90 du siècle dernier et dont témoignent en monde francophone autant la réception de la frame theory et les prolongements des thèses de Joas et de Honneth, que les réflexions d'un Cefaï sur l'action collective ${ }^{1}$, l'expérimentalisme démocratique constitue sans conteste une proposition forte. Celleci a permis de déplacer les lignes du néo-institutionnalisme en apportant une théorie de l'apprentissage centrée sur les effets possibles de déroutinisation des pratiques grâce au benchmarking et au co-design, notamment. L'idée-clé de Charles Sabel à ce propos, énoncée dès l'article de 1998 co-signé avec Dorf², est celle d'un apprentissage social soutenu par des procédures collectives de monitoring où les différentes tentatives de résolution de problème peuvent être co-évaluées et répercutées dans des ajustements continus grâce à des plateformes de focus-groupes. Cette idée largement développée par la suite est à la base d'un procédé de construction des décisions collectives qui permet de combiner à la fois les avancées du polycentrisme et de la polyarchie tels que les concevaient Ostrom et $\mathrm{Haas}^{3}$, tout en y apportant une conception de l'action collective renouvelée, indépendante des présupposés d'olson et intégrant tant les gains de la multiple choice theory $\left(\mathrm{Scharpf}^{4}\right)$ que ceux de l'approche des contrats incomplets à la façon de Willamson et de North ${ }^{5}$. Ce grand chantier a permis d'envisager une autre manière de concevoir la théorie de la gouvernance en la fondant sur une conception de la transformation du rapport aux normes interne au processus de l'action collective, et ceci grâce au recours à des dispositifs d'auto-évaluation et d'alignement des préférences sur le mode, en particulier, de la deliberative policy analysis ${ }^{6}$.

2 Cette manière de relier l'expérimentalisme démocratique dans sa version canonique énoncée par Sabel et directement rattachée à Dewey à un courant théorique plus général en théorie de la gouvernance est une façon de situer son impact dans un champ qui dépasse largement celui de la pensée juridique, comme le fait l'article co-écrit avec 
William Simon ${ }^{7}$. De fait, la théorie néo-institutionnaliste des normes a beaucoup gagné en acuité grâce aux déplacements effectués vers l'action collective et l'apprentissage par l'incorporation de l'approche expérimentaliste. Mais celle-ci n'a pu donner cette impulsion dans le domaine de la pensée juridique que parce qu'elle procédait de l'expérience de champs théoriques différents, comme une théorie sociologique des organisations au sein d'institutions privées mobilisant un nouveau type de gouvernement participatif, une psychologie du développement axée sur l'autotransformation des agents en cours d'action et une philosophie politique de l'action collective de type interactionniste et procéduraliste. Il n'est pas question de noyer la trace de la proposition expérimentaliste dans une vague mouvance partageant un ensemble de tendances communes dans un marché des idées évolutif, mais de comprendre la nature exacte de la combinaison théorique qui a rendu si attractive la proposition expérimentaliste. Cet effort de caractérisation épistémologique nous permettra aussi de formuler une hypothèse sur une éventuelle faiblesse de cette proposition face à un contexte perçu comme critique et justifiant des prises de décision dans l'urgence face des risques majeurs, qu'ils soient sanitaires ou financiers, par exemple.

Dans un premier temps, nous rappellerons donc d'abord les éléments déterminants de la proposition expérimentaliste dans ce que nous nommerons sa version restreinte, à savoir sa formulation par Sabel et sa reprise de Dewey ${ }^{8}$. Il s'agira d'éclairer cette formule canonique selon laquelle "experimentalist governance is a recursive process of provisional goal-setting and revision based on learning from the comparison of alternative approaches to advancing them in different contexts $»^{9}$. Dans un deuxième temps, nous tenterons d'attirer l'attention sur la portée et, éventuellement, la limite de la référence à Dewey pour assurer la dimension démocratique de cette politique expérimentaliste sur la base d'une certaine théorisation du rôle du public comme agent de ses propres intérêts. Dans un troisième temps, nous nous intéresserons à la version étendue de la proposition expérimentaliste, en particulier dans les formules cherchant à optimiser la participation et la production collective d'un savoir de l'action en cours de transformation, notamment dans le contexte de la gestion des risques. Dans un quatrième temps, nous tenterons d'identifier si des versions non standards ont été développées, consistant à sortir des conditions d'application stable du modèle en régime de révision des institutions démocratiques. Ces versions permettraient d'envisager aussi des circonstances où l'expérimentalisme serait convoqué au-delà des présuppositions récursives d'une société auto-législative ${ }^{10}$ de manière à faire face à l'impuissance avérée des tentatives d'auto-correction. Nous chercherons alors à comprendre, dans un dernier temps, si l'évolution éventuelle de l'expérimentalisme vers des formes non standards ainsi que vers une sortie plus radicale des pratiques expérimentalistes elles-mêmes ne proviendrait pas d'une faiblesse interne de celui-ci, insuffisamment identifiée et travaillée.

\section{Le cœur du procédé expérimentaliste}

Il faut distinguer deux niveaux dans l'approche des pratiques de gouvernance que propose l'expérimentalisme démocratique : il est constitué, d'un côté, par un certain état d'esprit ou par une philosophie de l'action démocratique et, de l'autre, par un réagencement des mécanismes d'action institutionnelle. 
5 Sur le plan de la philosophie de l'action, l'expérimentalisme s'appuie sur l'idée que l'action collective gagne en efficacité et en capacité d'anticipation quand elle élargit les possibilités de participation des différents acteurs concernés et qu'elle tire parti de la connexion plus intense qu'engendre cette participation entre l'engagement partagé et les apprentissages situés. L'action collective est ainsi conçue sur le mode d'un double loop learning qui permet aux informations locales de remettre en question la vision générale de l'action à réaliser et d'envisager l'intérêt éventuel de certaines déviations par rapport à un programme donné. Ce principe de circulation interne entre une volonté commune et l'ajustement possible en fonction d'informations incomplètes et inégalement distribuées, crée les conditions d'une communauté d'expérimentation récursive, soucieuse d'assimiler des motifs raisonnables de bifurcation provenant d'intérêts situés tentant d'appliquer des normes communes et susceptibles de fournir de nouvelles informations en continu ${ }^{11}$.

6 C'est en fonction d'un tel esprit qu'on peut envisager de manière plus précise, une série d'agencements institutionnels qui vont garantir effectivement les effets de cette volonté récursive d'apprentissage mutuel. Le principe qui prévaut, dans ce cas, est celui de responsivité institutionnelle : puisqu'un espace de transaction avec les contraintes contextuelles est admis, il est fondamental de mettre en place des mécanismes de détection des erreurs dans la transposition locale des règles communes, d'inciter un retour évaluatif des résistances et des ajustements imposés par les situations, de prévoir une réévaluation des orientations générales en fonction de ces nouvelles informations. Pour qu'un tel processus réflexif fonctionne, il faut que la coopération entre les niveaux d'action soit redéfinie de telle sorte que la hiérarchisation habituelle entre l'unité de coordination et les entités d'exécution soit modifiée dans le sens d'une plus grande horizontalité sur le modèle d'activités conjointes: il s'agit désormais de combiner une vue incomplète de l'ensemble du processus avec une expérience biaisée de ses potentialités en permettant à un reporting local le plus suivi possible d'accroître en même temps la masse d'informations disponibles, mais aussi la transparence nécessaire à leur traitement. La responsabilité à l'égard du processus est ainsi coconstruite en fonction d'une téléologie partagée, à savoir produire ensemble de meilleures solutions en cours d'action. La coopération responsable réside dans l'interaction entre l'obligation de reporting et la transparence dans le traitement de l'asymétrie d'information. La situation sous-optimale des processus décisionnels face à la diversité du réel est assumée à travers une tâche continue de révision alimentée par l'accroissement des informations fournies par les apprentissages situés (récursivité).

7 Toutefois, Sabel est aussi conscient des limites qui peuvent apparaître face à cette vision ingénue de la coopération sociale, rendant certainement possible dans des espaces dédiés - comme des zones d'activité industrielle créant de bonnes conditions d'interaction ${ }^{12}$ - de bonnes pratiques d'échanges de savoir-faire réduisant les coûts de transaction et améliorant constamment la recherche de solution. La condition implicite de ces expérimentations réalisées à la fois dans une proximité géographique, mais aussi dans une proximité de métiers et d'objectifs liés à un secteur de production, est de s'appuyer sur une sorte de tissu communautaire résultant à la fois du partage de valeurs épistémiques et de valeurs institutionnelles. La fiabilité d'un partenaire local réside dans la mémoire des expériences antérieures et dans leur interprétation commune en fonction de cadres épistémiques partagés. Dans une telle situation, l'élargissement $\mathrm{du}$ socle coopératif au-delà d'une histoire partagée en fonction 
d'opportunités locales peut s'avérer particulièrement difficile, parce que les conditions de la confiance ont été supposées données. On pourrait donc opposer à une tendance coopérative facilement stimulable dans de bonnes conditions de localisation, une tendance à la défiance, renforcée par l'avantage de la localisation qui amène à incorporer des barrières communautaires limitant les capacités d'échange à l'égard des étrangers et des nouveaux venus. L'absence d'attention au rôle joué par les conditions implicites de la coopération en contexte favorise leur répétition plutôt qu'une remise en question et un déplacement à l'égard de leur emprise tacite. Quand il est manqué, l'effet de proximité qui soutient le développement d'une coopération ne permet pas d'anticiper la mise en place d'un processus de répétition à l'insu des apprentissages réalisés. De fait, malgré la recherche de solutions partagées, l'échange de savoir-faire et le partage d'objectifs, voire la comparaison et le co-design, le fait de partager des représentations communes tacites de ce qui se passe engendre des sortes de routines interprétatives qui confirment les croyances fondamentales d'un secteur professionnel et des métiers qui le structurent. Les apprentissages pourtant « capacités » et renforcés viennent ainsi progressivement compléter une culture épistémique existante qui se maintient dans l'implicite et augmente son potentiel défensif face à la nouveauté ou à l'inconnu.

8 Dans bien des cas, l'histoire nous a appris que la solution à ce genre de limitation des informations nouvelles, dans un milieu fortement agrégé par ses pratiques, est d'en référer au rôle d'une norme extérieure - telle une agence administrative de contrôle capable d'inciter à la prise en compte d'autres représentations des contraintes de développement. Ces dernières résultent de représentations portées par d'autres métiers et d'autres secteurs professionnels et obligent à interrompre les routines interprétatives qui décidaient d'un idéal de performance. Mais cette manière de procéder n'est, du point de vue des apprentissages à réaliser, qu'une solution très partielle parce que, de son côté, elle suppose qu'il s'agirait uniquement de corriger une asymétrie d'information et d'élargir le socle de connaissances disponibles pour choisir les meilleures solutions. Or le problème est nettement plus complexe. Si d'un côté, l'implicite des barrières communautaires fait apparaitre un risque de répétition d'un savoir tacite jamais vraiment remis en question malgré les apprentissages expérimentés, une seconde limite du savoir coopératif apparaît quand il s'agit de lever cette barrière. La question de fait n'est pas simplement de sortir d'une idée de complémentarité permettant une amélioration continue des pratiques partagées pour passer à une idée de supplémentarité qui garantirait d'incorporer de nouvelles informations et d'étendre un premier socle d'évidence épistémique à un niveau plus avancé, moins dépendant de ses ancrages et plus à même d'incorporer les contraintes d'un contexte global. La nouvelle limite qui apparaît est celle d'une discontinuité et d'une déstabilisation de ce rapport primaire aux croyances fondamentales. Il ne s'agit pas simplement de les réviser et de les adapter en les faisant monter en généralité, comme dans une séquence binaire de type on/off, ignoré/connu. L'obligation engendrée par la prise en compte de nouvelles informations est en même temps l'exigence épistémique de se confronter à un régime d'incertitude des informations disponibles et donc de modifier le rapport aux croyances qui jusqu'à présent rendait possible l'accroissement des capacités à travers les apprentissages coopératifs. La prise en compte de réalités et de pratiques qui sont expérimentées par d'autres secteurs professionnels et par d'autres métiers, le fait de se confronter avec des cultures 
professionnelles différentes et des partenariats inhabituels exige ni plus ni moins que l'interruption de la répétition.

9 C'est à ce niveau qu'intervient plus précisément la proposition d'institutions pragmatistes capables d'orienter l'ensemble des acteurs concernés vers des pratiques expérimentalistes. Les enjeux d'apprentissage ou de « capacitation » doivent donc être correctement jaugés en fonction du problème qu'ils tentent de résoudre. Ils n'ont en fait pas grand-chose à voir avec la stimulation locale de capacités coopératives, même si celles-ci permettent de comprendre ce qu'il en est du potentiel d'apprentissage que peut contenir une interaction basée sur la confiance et la construction de nouveaux savoirs. Ils ont plutôt à voir avec le risque qu'encourent de tels apprentissages quand ils sont mis en œuvre sans prêter attention aux barrières communautaires qui les guettent lorsqu'ils s'adaptent à un secteur d'activité déjà constitué. Quand ce risque est pris en considération, on comprend que le niveau d'intervention des institutions pragmatistes essaie de combiner deux plans essentiels : d'un côté, le plan du rapport aux croyances ; de l'autre, le plan du rapport aux normes. Sur le premier plan, l'enjeu est de sortir de l'idée que la seule limite à combler est celle du biais d'informations pour s'assurer de meilleurs résultats. Sur le deuxième plan, l'idée est de remettre en question l'effet escompté d'une hétérorégulation garantissant une transformation des capacités par le seul effet de la commande et du contrôle externes. Dans l'action d'apprentissage visée par une institution pragmatiste, application des normes et production de savoir marchent de pair. Le passage à une action en régime d'incertitude n'aurait aucun sens si le vide créé par l'interruption des habitudes interprétatives était remplacé par des dispositifs normatifs éliminant tout doute dans la recherche de solutions. Les normes elles-mêmes doivent ainsi paraître dans leur dépendance de sentier par rapport aux engagements locaux et aux réévaluations qu'ils sont en mesure de suggérer. On est dans un processus de prise de décision orienté vers le long terme où des effets de révision sont d'emblée incorporés au choix et à la mise en œuvre institutionnelle des normes.

\section{Dewey et les présupposés du pragmatisme}

Cette conception de l'institution pragmatiste est directement redevable à Dewey et, plus particulièrement, à sa volonté d'articuler la démocratie comme totalité institutionnelle avec sa forme de vie particulière, la société civile, toujours locale et constituée d'intérêts situés. Si la remise en question des routines interprétatives et donc des croyances fondamentales est si essentielle dans sa conception des réformes de la démocratie, c'est précisément parce qu'elle s'appuie sur un processus d'enquête partagée qui met en relation les preneurs de décision et leur public. Un risque existe toujours dans une totalité sociale de perdre de vue les intérêts locaux, minoritaires, face à l'émergence d'agents dotés d'un impact global sur la société. Gouverner pourrait se résumer à éviter l'emprise monopolistique de ces agents et à garantir leur interaction avec un ensemble d'obligations publiques. Mais un tel partenariat privépublic met entre parenthèses le processus d'information permanente des politiques de l'intérêt public par la constitution de publics porteurs des demandes des communautés locales. L'approche macroscopique de l'intérêt public doit être provoquée en permanence par l'approche microscopique des attentes communautaires, l'identification de nouveaux besoins et l'apparition de nouvelles sensibilités. Reste alors 
à savoir comment un tel processus d'auto-attention de la société à elle-même peut être garanti, sans être simplement réduit aux effets incertains d'une évolution par sélection naturelle. La formation de l'opinion par l'art et les médias, l'élargissement de la participation par les mécanismes électoraux offraient certes des perspectives favorables dans les années $20^{13}$. Cette idée de l'espace public moderne est parfaitement résumée par Anderson ${ }^{14}$ qui estime que les joutes électorales périodiques et les manifestations publiques ont un effet de feedback sur la vie sociale qui enrichit le spectre des discussions publiques entre votants par la diversité des informations et des cadres épistémiques mobilisés, garantissant ainsi un effet d'apprentissage collectif ${ }^{15}$. Mais ces grandes institutions constitutives de l'espace public et de son potentiel réformateur comptaient surtout sur un effet de généralisation d'une culture de l'évaluation des intérêts particuliers par la vertu du débat et des procédures décisionnelles d'ajustement de l'ordre global. Il leur manquait cette dimension " pragmatiste " qui amène l'auto-attention de la société à se confronter avec sa propre fragmentation pour décliner des solutions adaptées aux multiples situations et se laisser inspirer par elles dans sa vision plus générale. A cet égard, Sabel rappelle cette idée de subdiviser les comtés de Virginie en une multitude de petites assemblées qui seraient autant de petites républiques dotées de différents pouvoirs d'administration ${ }^{16}$. Ce changement d'échelle fait partie intégrante de l'institution pragmatiste : il ne s'agit pas simplement de prôner le subsidiarisme au nom d'une certaine vision de la totalité sociale avec son ordre hiérarchique interne et solidaire, il ne s'agit pas d'un problème de délégation de pouvoir et d'allocation de ressources, mais de reconnaître un ordre d'agrégation sociale spécifique, qui s'effectue par le bas, dans les choix de proximité. L'agrégation sociale ne se produit pas exclusivement et prioritairement par en haut, par élimination et limitation des attentes locales, elle s'effectue au contraire par l'apprentissage de la combinaison et de la redéfinition de ces attentes en fonction des collaborations localement choisies. Dans ce sens, chez Dewey, les intuitions d'Henry Georges dans Poverty and Progress sont venues compléter et déplacer les perspectives du Public et ses problèmes ${ }^{17}$ en donnant la prépondérance aux processus de proximité dans les institutions pragmatistes, là où les grands mécanismes de l'espace public moderne semblaient dans un premier temps pouvoir garantir l'auto-attention nécessaire de la démocratie.

11 Il n'en reste pas moins que certaines questions demeurent en fonction des perspectives ouvertes dès Le public et ses problèmes. Ce public local et communautaire auquel aspire Henry Georges, procédant à l'ajustement des métiers dans un esprit de coopération et accumulant ainsi un savoir nouveau dans une dynamique d'échange et d'apprentissage, - ce public n'a pas seulement une propension à conserver son environnement communautaire contre de nouveaux entrants, comme le souligne Sabel pour parfaire sa proposition $^{18}$, mais il est surtout supposé posséder des capacités d'auto-gouvernement en fonction de la promotion de ses intérêts et de l'accumulation de ses nouveaux savoir-faire.

Or, c'est précisément cet aspect des choses que Le public et ses problèmes abordait déjà, notamment en rapport aux conditions que requiert une enquête sociale pour aboutir à des résultats probants. Joëlle Zask en souligne trois ${ }^{19}$. Pour que le public puisse devenir en quelque sorte l'enquêteur de ses problèmes, il doit être capable d'identifier les problèmes qui l'affectent, de déterminer qui en est responsable dans l'organisation sociale et quelles règles de la vie en commun sont concernées. Il doit enfin être capable 
de critiquer ces mandataires et de revendiquer une mise sous contrôle de leur action. C'est pourquoi il semble alors évident pour Dewey que la contrepartie nécessaire à une telle action du public sur ses problèmes réside d'abord dans le système éducatif luimême. Les propositions de Dewey concernant l'enquête sociale s'appuient sur les capacités d'un public instruit et donc sur un système démocratique dont une fonction majeure est d'assurer l'accès à une éducation citoyenne pour tous de manière telle que le droit d'exercer son jugement soit non seulement garanti, mais aussi supporté par le concours des libertés publiques d'accès à l'information, à l'instruction et au débat grâce à la liberté d'association des personnes. En fait, l'auto-attention ne rend pas un public actif: elle suppose un public capable de dégager des connaissances suivant une méthode qui le soustrait à sa situation de spectateur et l'amène à poser les jugements nécessaires pour transformer les conditions qui l'affectent.

La nuance qu'apportent les considérations de Zask réside dans l'extension nécessaire de la réflexion sur les préconditions de l'expérimentalisme démocratique. Il est de fait démocratique non pour des raisons substantielles liées à la nature de ses recommandations en termes de coopération, d'échanges de pratiques, voire de confiance ou de respect mutuel dans la co-construction des solutions. Il est "démocratique " parce qu'il inscrit son processus d'expérimentation sociale dans un environnement institutionnel déterminé qui est celui des démocraties modernes construites sur la culture de la recherche de solutions communes à travers la production de consensus argumentés. Dans ce sens, l'expérimentalisme n'a pas de fonction instituante, mais au mieux une fonction constituante, qui présuppose un horizon déjà culturellement établi quant aux objectifs à atteindre ainsi que sur la manière d'y arriver. Sa fonction essentielle consiste à lever les obstacles et à renforcer la productivité de la coopération en activant des solutions procédurales. Mais ces solutions s'inscrivent dans un espace culturel déjà acquis: elles comptent sur une volonté d'influencer les situations problématiques qui contrecarrent les intérêts d'une communauté. Dans ce cas, le pouvoir d'influence est déjà établi, de même qu'un certain pouvoir de diagnostic et de coopération. C'est pourquoi, comme l'écrit Zask, l'enquête est d'abord à considérer, chez Dewey, comme une « posture coexistante à la vie $»^{20}$ :

De l'habitude à l'enquête, en passant par les expériences ordinaires, il n'y a aucun saut ontologique : l'interaction entre un individu et son environnement assure en même temps la continuité de l'existence, l'adaptation du sujet aux situations qu'il rencontre et l'exercice d'une influence sur ces situations de sorte qu'il puisse rétablir sa conduite ${ }^{21}$.

Dès lors, le public est acteur et peut renforcer, voire rétablir ses capacités d'influenceur de son milieu de vie, parce qu'il s'est constitué dans des institutions sociales et politiques qui lui ont donné accès à des ressources cognitives lui permettant d'identifier des problèmes communs et de les relier à différentes fonctions ou degrés de responsabilité dans l'organisation de sa communauté22. Si on suit cette analyse complémentaire qu'apporte la lecture de Zask, on comprendra que l'expérimentalisme doit s'étendre à une prise en compte plus systématique des préconditions de son propre procéduralisme. Même si l'on peut considérer avec Dewey qu'un public moderne devrait être un public d'enquêteurs par l'effet même de son appartenance à un milieu institué sur les vertus du civisme démocratique, il n'en reste pas moins que la formation de ses capacités d'action collective et d'analyse institutionnelle ne peut être supposée donnée et doit venir renforcer, à titre de précondition, les effets attendus de l'expérimentalisme que prôneraient des «institutions pragmatistes». La seule 
référence à un système éducatif et la connaissance pratique de la distribution des responsabilités dans un système de mandataires ne suffisent pas à garantir ces préconditions, comme on aurait encore pu le croire à une époque où s'instaurent les principes d'éducation publique, de suffrage universel et de libre association. Tout le problème réside de fait, comme le souligne Zask, dans la rencontre d'« objets prétendument indépendants de nos expériences ", "inexpérimentables ", qu'il faut parvenir à « modifier de sorte qu'ils se prêtent à la poursuite de nos expériences »"23.

\section{Vers un « expérimentalisme étendu »}

15 Sur ce plan, l'« expérimentalisme étendu » défendu par l'analyse délibérative d'auteurs comme Wagenaar ${ }^{24}$, Booher ou Innes ${ }^{25}$ nous paraît particulièrement significatif. Leurs propositions s'attaquent précisément aux préconditions de l'expérimentalisme restreint envisagé par Sabel et qui compte obtenir un effet de renforcement des capacités d'action uniquement à partir des procédures qu'il propose, tant sur la manière de les gouverner et de les articuler avec un souci de généralisation que sur la manière de les stimuler à la base par leur gain en efficacité et en autonomie. Un aspect fondamental de l'analyse délibérative est de créer les conditions de croisement entre des groupes dont les cultures d'action divergent ou s'ignorent. Un tel type d'apprentissage mettant en concurrence des régimes de signification et d'interprétation hétérogènes ne peut se mettre en place sans une attention épistémique de nature spécifique ${ }^{26}$. Même si des problèmes peuvent a priori être considérés comme communs, ces groupes n'en comprennent ni les raisons ni les enjeux de la même manière. Il ne s'agit pas simplement de marier des compétences différentes et des métiers différents autour de procédés de production ou de transformation dans une zone d'activité industrielle, il s'agit de se confronter collectivement à un problème qui à la fois entrave et semble en même temps échapper au contrôle, telles par exemple une maladie incurable, des allergies mal définies, des contraintes environnementales encore mal maîtrisées au niveau du développement futur d'un territoire, etc. Dans ce cas, un certain nombre de préconditions épistémiques et pratiques interfèrent avec toute tentative de procéduralisation expérimentaliste. Il est nécessaire que puissent émerger, comme le suggèrent Innes et Booher ${ }^{27}$, non seulement de nouvelles significations, mais aussi de nouvelles heuristiques. Il ne s'agit pas uniquement d'argumenter et de contre-argumenter, mais de produire un milieu tiers de compréhension mutuelle et, dès lors, d'extension des solutions pratiques possibles.

Un déplacement de ce genre ne relève pas simplement d'un jeu sémantique qui consisterait à apprendre de nouvelles désignations des problèmes rencontrés. En envisageant ce processus de sortie des habitudes interprétatives, les auteurs de l'analyse délibérative posent un problème plus fondamental, à savoir celui du lien entre les significations habituelles et l'identité des groupes qui les véhiculent. Dans le cadre du déplacement vers un milieu tiers de nouvelles significations, il est nécessaire d'anticiper aussi une mise en question des repères identitaires et, donc, de saisir l'importance de mobiliser des compétences susceptibles de traverser l'écart inévitablement créé par le retour de ces nouvelles significations sur une forme de non identité à soi désormais indispensable à l'extension des possibles (les nouvelles heuristiques). On ne peut, dans une dynamique de ce genre, faire fi des questions que pourraient poser d'éventuelles réactions défensives et la volonté de bloquer les 
nouvelles significations au profit de la répétition des anciennes. Ainsi, quand Innes et Booher parlent de créer une "communauté d'enquête ${ }^{28}$, ils la lient explicitement à une culture fondamentale qu'ils ont d'abord garantie pour elle-même à titre de précondition, à savoir la culture du dialogue. C'est l'apprentissage des règles du dialogisme qui pourra guider l'élargissement des possibles dans une communauté d'enquête. S'il est toujours bien question de "learning by doing ${ }^{29}$, une théorisation des préconditions est néanmoins nécessaire pour garantir le succès d'un tel processus par rapport aux tensions qu'il ne manque pas de rencontrer et qu'il pourrait être tenté d'exclure précisément de son champ d'influence. Il est impossible d'accepter des contradictions, de prendre en compte des ambiguïtés, voire des volte-face, de jouer avec des asymétries d'information et des rapports ambivalents à l'autorité en charge d'une question, sans avoir préalablement défini des lignes de conduite et travaillé spécifiquement sur elles pour s'y tenir dans le processus.

Pour y arriver, les auteurs proposent de mobiliser la DIAD Theory ${ }^{30}$ de manière à former les acteurs concernés au processus collaboratif pour maintenir son orientation dialogique. Ils identifient des préconditions de trois ordres: sur le plan de la participation, sur le plan de l'interaction et sur le plan de l'apprentissage. C'est ce qui définit plus précisément l'expérimentalisme lorsqu'il est étendu aux préconditions de l'action procédurale attendue des institutions pragmatistes. Sur le plan de la participation, l'extension de l'expérimentalisme porte sur la nécessité de reconstruire les intérêts en présence, non seulement selon leur diversité, mais aussi pour expliciter leur interdépendance. Sur le plan de l'interaction, l'enjeu de l'extension consiste non seulement à rappeler les conditions élémentaires de la communication sans contrainte de Habermas, mais aussi à valoriser la qualité de l'engagement (membership) dans un processus dialogique comme produisant un type de responsabilité collégiale ou partagée à l'égard de son aboutissement. Enfin, sur le plan de l'apprentissage, l'ensemble du processus doit être abordé de manière téléologique, et pas uniquement réflexive, en tant qu'il oriente dans une démarche de transformation d'une situationproblème à partir de nouvelles solutions ou hypothèses d'action à porter collectivement, donc susceptibles potentiellement de redéfinir les rôles de chacun. L'idée est ainsi de travailler à mettre en œuvre un processus d'explicitation en trois étapes : l'explicitation de l'interdépendance des intérêts passe par l'explicitation de la force collégiale du processus pour aboutir à l'explicitation d'une téléologie orientéeacteur autant qu'objet, c'est-à-dire tentant de combiner solution innovante et redéfinition des rôles.

Une autre manière de formuler cette extension de l'expérimentalisme à ses préconditions est de concentrer l'effet recherché sur une opération majeure où il s'agit fondamentalement de mettre en résonance trois plans nécessaires d'apprentissage pour garantir le succès de l'expérimentalisme. La nouveauté de cette version qu'on trouve à notre sens dans le triangle éducatif de Hartmut Rosa ${ }^{31}$ ne réside pas tant dans l'appréhension de ces différents plans de précondition comme susceptibles de faire face à l'urgence qui a saisi les institutions démocratiques et déstructuré leur rapport au temps, que dans la désignation de l'opération d'articulation de ces plans qui n'est pas pensée comme telle chez Innes et Booher. Ce qui trouve ainsi à se déployer chez Rosa, c'est ce que Sabel nomme déjà l'attention à l'attention et qu'Innes et Booher développent dans leurs préconditions de réalisation à travers une sorte d'attention préalable pour susciter cette réflexivité sociale. Mais ce qui est mis entre parenthèses dans les deux cas, c'est ce que l'opération tente effectivement de produire, à savoir un 
autre type de rapport à l'attention. On dira avec Rosa que l'enjeu recherché tant par l'expérimentalisme restreint que par l'expérimentalisme étendu, c'est de parvenir à une mise en résonnance de l'attention avec ce qui affecte son expérience de manière à le rendre disponible autrement ou à le laisser advenir autrement ${ }^{32}$.

L'idée d'un processus éducatif compris sur le modèle musical d'un accord entre différentes tonalités ${ }^{33}$, voire selon la métaphore de la concordance entre des ondes sonores, attire surtout l'attention sur deux aspects des préconditions de l'expérimentation sociale vécue comme situation d'apprentissage. D'une part, structurellement, un milieu se constitue par un effet de triangulation, qui dépasse le modèle interactionniste: le champ d'apprentissage se co-construit à partir des positions asymétriques de la relation pédagogique ; pouvoir découvrir ensemble, c'est à la fois s'autoriser à découvrir et désirer le détachement de soi de celui avec qui on découvre ${ }^{34}$. D'autre part, pragmatiquement, chacun des pôles impliqués dans ce milieu de résonance est touché dans son rapport à soi par la participation au processus en cours. Il évolue dans sa disposition à être atteint par l'intérêt partagé pour un thème. En identifiant ainsi plusieurs conditions pour garantir cette affection commune qui devrait parcourir le processus éducatif, Rosa parvient à expliciter davantage un présupposé de l'expérimentalisme social étendu: l'objet visé n'est pas une simple donnée matérielle ou sociométrique à laquelle il faudrait appliquer une méthode pour renforcer le potentiel de résolution collective des problèmes par les agents concernés. Cet objet-problème est constitutif d'un champ d'action qu'il permet de mobiliser et dans lequel sa position-tiers à l'égard des parties concernées va avoir une double incidence: celle d'un révélateur de nouvelles possibilités conditionnées par la coopération avec d'autres et celle d'un ajustement du rapport à soi nécessaire pour mettre en œuvre cette coopération. Dans les deux cas, la médiation de l'objet est décisive : c'est en étant affecté par ce qui n'est pas encore expérimenté et en se laissant toucher aussi par cette affection chez l'autre à l'égard de ce qui pourrait être expérimenté ensemble que se constitue un espace capacitateur d'un véritable expérimentalisme. Il faut donc en revenir à cette idée exacte, mais insuffisamment déployée dans l'idée de public deweyen, de la rencontre d'«objets prétendument indépendants de nos expériences $»^{35}$, qu'il faudrait parvenir à « modifier de sorte qu'ils se prêtent à la poursuite de nos expériences $»^{36}$. Le jeu externalité/internalité ne permet pas de rendre compte entièrement de l'opération qui est exigée par l'expérimentalisme : aucun processus ne parvient à internaliser des problèmes par la capacitation de mécanismes coopératifs, s'il ne maintient pas un effet propre à la contrainte externaliste qui consiste à mettre hors-jeu les capacités habituelles du jeu internaliste. Dans ce cas, ce qui doit être garanti pour compter sur une plus-value expérimentaliste, ce n'est pas uniquement une adaptation ou un réajustement des capacités, mais de manière radicale une transformation de leurs préconditions, c'est-àdire une transformation du rapport de croyance que les acteurs entretiennent avec elles et qu'ils ne peuvent mettre en cause sans la médiation d'un tiers susceptible de les " toucher ", donc de modifier leurs affects ou leurs certitudes.

20 Il est donc question ni plus ni moins d'un certain ébranlement du rapport au champ d'action, comme une précondition de l'expérimentalisme : une confiance en soi et en l'autre qui va au-delà de ce qui est attestable devant une situation-problème et qui n'adviendra jamais qu'en fonction d'un déplacement et d'un partage des affects suscités par cette situation hors de l'expérience habituelle. Il est ainsi possible, souligne de nouveau Rosa ${ }^{37}$, qu'en acceptant une certaine prise de risque selon les représentations 
standards, qu'en admettant d'explorer la perte de contrôle garanti par un cadre prédonné d'action, on parvienne, en comprenant ce qui se joue ainsi, à élargir le champ des possibles d'une manière très spécifique, à savoir répondre à l'impuissance du contrôle par les moyens et les fins disponibles en expérimentant un champ d'action où ils sont rendus indisponibles. Ce n'est pas dès lors la maîtrise de l'action qui permet de rendre un monde disponible ou de trouver des solutions, mais c'est d'abord de partager la manière dont un monde se donne dans son indisponibilité qui permet d'envisager des actions qui préparent à ce réel et permettent de se laisser interpeller par luij ${ }^{38}$.

\section{Un expérimentalisme non standard : la « sociologie des absences »}

Ces différents aspects mis en évidence avec les réflexions de Rosa nous amènent à la limite la plus radicale de ce que nous avons nommé l'expérimentalisme standard étendu. Les préconditions mises en évidence avec les tenants de la Deliberative policy analysis et l'opération de mise en résonnance avec l'indisponibilité d'un monde à expérimenter telle que la conçoit Rosa concernent des processus de résolution de problème et d'apprentissages sociaux situés dans un horizon où l'efficacité des mécanismes participatifs et délibératifs demeurent la base de l'expérience commune, comme dans la société bien ordonnée de Dewey ou de Rawls ${ }^{39}$. Mais d'autres approches focalisées sur les études critiques et, en particulier, sur les épistémologies subalternes revendiquent une conception de l'expérimentalisme qui ne se limite pas à créer les conditions d'institutions pragmatistes susceptibles de favoriser des modes plus coopératifs de résolution des problèmes en démocratie. Pour elles, cette posture d'enquête partagée peut aussi s'appliquer de manière non standard à des éléments qui semblent d'emblée se soustraire à l'expérience commune, au point de ne se signaler que par leur absence.

22 L'argument ne porte pas ici sur la question de savoir si le pragmatisme permet d'envisager le consensus expérimentaliste sans le présupposer comme préexistant ${ }^{40}$. $\mathrm{Au}$ contraire, c'est la définition même du pragmatisme qui l'amène à considérer la condition de possibilité comme interne à l'effectuation. Elle n'est en aucun cas présupposée, mais s'élabore et se révise à l'intérieur du processus de sa construction sociale, à la manière d'un horizon constamment renouvelét ${ }^{41}$. Suivant cette perspective, une certaine conception de la démocratie inclusive ${ }^{42}$ tentait déjà d'aller au-delà d'un expérimentable standard pour incorporer des intérêts secondaires ou minoritaires dans la délibération ${ }^{43}$. De même, la volonté d'étendre l'expérimentalisme au-delà de catégories ontologiques habituelles de façon à incorporer de nouveaux sujets de droit dans l'espace délibératif sont autant de tentatives d'élargissement fonctionnel de l'expérimentalisme qui sont susceptibles d'amener à identifier des préconditions supplémentaires. C'est le cas, par exemple, des porte-parole qui peuvent donner voix aux intérêts d'actants non humains et les introduire dans un processus inédit d'expérimentation de normes.

23 Mais l'expérimentalisme non standard apparaît dans des contextes plus radicaux quand il ne s'agit pas uniquement d'explorer les préconditions d'un imaginaire social en fonction, par exemple, d'un non-vivant, échappant à l'ordre culturellement prédéterminé des êtres sensibles ${ }^{44}$. L'enjeu devient crucial quand le travail de l'autoattention sociale revient à la question de l'indisponibilité du monde et de 
l'expérimentation de l'inexpérimentable, comme à une situation de base, nécessaire pour entreprendre toute action. Dans cette optique, toutes les recommandations pensées en termes d'optimisation institutionnelle pour renforcer les capacités d'engagement des publics et les processus d'ajustement des décisions institutionnelles sont dépassées. Il faut dès lors sortir des cadres techniques auxquels se limitent les approches de type institutionnaliste, focalisées sur la réduction des coûts de transaction engendrés par les relations entre des institutions établies et les incertitudes de leurs publics. Roberto Unger suggère même que c'est l'application non standard de l'expérimentalisme démocratique qui est son moteur le plus probant. Il ne s'agit pas simplement d'accroître la réflexivité de parties prenantes dans des processus garantissant à la fois une plus grande symétrie des informations, plus de transparence et plus de révisabilité, mais d'abord de produire cet écart dans les pratiques en cours qui permet une disposition favorable au changement ${ }^{45}$. Dans ce cas, l'expérimentalisme concerne aussi les aspects critiques des institutions démocratiques, leurs impasses ${ }^{46}$, et il prétend que ses propositions sont susceptibles de transformer l'impuissance des structures conventionnelles ${ }^{47}$. Comment un tel saut est-il concevable au-delà d'une simple démarche incantatoire à l'égard des ressources de l'imagination créative que libèrent la coopération sociale, la fusion d'horizons mis en résonnance et la production d'institutions plus réactives?

Le pivot des applications non standard de l'expérimentalisme réside dans la reprogrammation de son usage social. En éclairant la référence à Dewey, Zask a montré que le rapport à l'inexpérimentable était un élément décisif de la démarche cognitive à la base de l'expérimentalisme démocratique. En créant un milieu d'apprentissage et de coopération entre différents publics, les institutions démocratiques permettent de retrouver une influence sur les problèmes et d'envisager des modes de résolution nouveaux de ceux-ci. L'enquête est dans ce sens un moment de dépassement de l'inexpérimentable pour sauter un obstacle et relancer la poursuite ordinaire des intérêts. Mais en même temps, le blocage et le risque que manifeste le caractère inexpérimentable de certains problèmes ont une valeur plus radicale que celle d'un problème indiquant des options sous-optimales et insatisfaisantes, comme autant de points de vulnérabilité à internaliser. L'inexpérimentable peut aussi désigner des situations d'échec et d'aveuglement, donc des situations qui échappent au contrôle ou sont éventuellement déniées, voire neutralisées, en tant que hors sens pour l'action d'un groupe ou d'une communauté. Alors que tout le pragmatisme standard (restreint ou étendu) mobilise sur des capacités supposées à stimuler, inciter ou renforcer, l'expérimentalisme non standard s'intéresse pour sa part aux incapacités, conscientes ou refoulées, qui constituent le hors sens d'un milieu d'action. Il envisage donc d'agir sur le manque ou l'absence de capacités, le refus de coopérer et de co-construire de manière à interrompre l'accoutumance à cette impuissance en tentant de s'y rapporter différemment. Dans ce cas, l'expérimentalisme prétend s'établir à la limite de l'horizon d'un pouvoir démocratique donné et entend poser des conditions d'action qui transformeraient le rapport à ce qui est hors d'atteinte de ce pouvoir. Le geste semble inclusif, comme celui de l'enquête deweyenne quand elle ramène dans le champ des capacités d'action une situation-problème insatisfaisante, mais dans ce cas, il ne s'agit pas d'inclure à des capacités données, mais de faire surgir par le rapport au tiers exclu lui-même un autre rapport aux capacités supposées.

Cette ressource pragmatique de l'expérimentalisme n'apparaît réellement qu'avec les versions non standard. C'est en se confrontant à la situation d'exclusion de 
l'inexpérimentable, hors sens pour les capacités données, qu'un autre rapport aux capacités mobilisables peut prendre forme. Il n'est pas uniquement question de coopération, d'apprentissage réciproque, de confiance et d'auto-correction des procédures de décision, mais aussi d'auto-aveuglement inhérent à tous ces mécanismes d'action collective, voire de répétition du manque sans qu'il s'agisse d'un simple effet de routinisation et d'habitude interprétative voire, encore, de conservation des normes standards de transformation et de révision, en fonction des arbitrages du commun reliant un espace d'activité et un ordre institutionnel supposé le garantir.

Quand il redéfinit l'expérimentalisme dans cette perspective non-standard, Boaventura de Sousa $\operatorname{Santos}^{48}$ le reconstruit d'abord théoriquement, en fonction du statut particulier qu'y prend le tiers exclu, à savoir les subalternes ou les absents. L'intérêt de cette "sociologie des absences $~^{49}$ réside surtout dans le travail sur l'absent qu'elle désigne et dont elle cherche à modifier le rôle imposé par le savoir hégémonique. Dès que l'impuissance à construire des solutions sans réduire constamment la multiplicité des histoires et des situations est prise en compte, ce qui devient capital ce n'est pas tant d'identifier le mécanisme fondamental qui permet de maintenir une telle illusion ou de la refouler, mais c'est de saisir précisément ce qui se livre à l'expérimentation dans un rapport dès lors inédit et ce qui survient ainsi comme objet d'enquête à l'insu de l'entreprise déconstructrice des évidences hégémoniques. Comme le signale Santos, ce sont ni plus ni moins des inexistences qui viennent à interférer avec les procédés habituels de résolution des problèmes. Les "parties disqualifiées des totalités homogènes $\|^{50}$, considérées comme non pertinentes, sont désormais ciblées comme susceptibles de déployer une autre échelle de possibles. Dans ce sens, était-il possible sans un tel expérimentalisme non standard de s'intéresser aux savoirs naturels, de convoquer l'expertise quotidienne de riverains ou d'autres groupes concernés de la société civile, comme des patients par exemple en institution de soins, d'évoquer des solutions communautaires et négociées à des problèmes présentés comme relevant de l'ordre public? Plus radicalement encore, n'est-ce pas un expérimentalisme non standard qui permettrait de prendre appui sur des groupes discriminés, sur des associations de victimes, ou sur des associations d'activistes, pour chercher des solutions originales et de nouvelles voies d'action?

L'élément-clé de ce front expérimentaliste non standard réside dans le rôle dévolu au tiers exclu comme condition d'un autre rapport aux capacités d'action. Alors que le pragmatisme qui sous-tend l'expérimentalisme standard privilégie des capacités déjà données d'auto-transformation des communautés politiques habituées à se confronter à des échanges d'idées et de pratiques, tout en sauvegardant l'idée d'un équilibre possible des différents intérêts, l'expérimentalisme non standard met en cause l'échec à envisager un rapport plus critique aux capacités et à considérer aussi leur rôle dans la reproduction d'équilibres préconçus. Les capacités de coopération et de coconstruction sont bien les mêmes qui entretiennent les processus d'exclusion et de discrimination, à partir du hors sens des intérêts absents dans leur forme de vie. Cette équivoque de la capacité, en même temps source d'impuissance, est le centre de l'expérimentalisme non standard qui lui permet de s'appliquer aux situations de crise et de contraction de la totalité sociale sur ses évidences. Et pour saisir la capacité dans son impuissance, il faut partir de ceux/celles qu'elle exclut d'emblée de son milieu de coopération pour envisager précisément un autre rapport à cette capacité. L'impuissance est l'insu de l'activisme participationniste et institutionnaliste favorisé par l'expérimentalisme standard. Ce dernier concentre l'attention sur les gains en 
réflexion réalisés par les acteurs mobilisés, mais il perd d'autant plus de vue ceux qui demeurent absents de ces scènes de convocation.

On pourrait ainsi penser, en fonction d'un expérimentalisme standard, qu'il soit restreint ou étendu, qu'aucune institution pragmatiste ne pourrait aider des communautés locales à se confronter à des problèmes qui restent pour elles inexpérimentables parce que hors sens (comme le génocide) ou hors mesure (comme la destruction du milieu naturel ancestral). Pourtant, la démarche de l'expérimentalisme non standard consiste à déplacer l'axe de convocation des parties prenantes pour qu'un autre pouvoir de convocation puisse s'affirmer, celui du hors sens ou d'un "signifiant vide ", qui devient ainsi le signe de l'absence problématique de capacités à prendre en compte. On peut partager autre chose que le savoir ou le pouvoir. Certains auteurs parlent d'une autre manière de " ressentir $»^{51}$, d'autres encore d'une contre-pédagogie ${ }^{52}$ et d'un détachement (delinking ${ }^{53}$ ) à l'égard d'un ordre épistémique donné et donc à l'égard de ce qui est conçu comme ne comptant pas ou comme étant impossible à comptabiliser. On se rend compte alors que d'autres institutions éducatives et culturelles, mais aussi de justice sont nécessaires pour qu'un travail de résolution de conflit, par exemple, puisse s'imposer comme une condition expérimentale pour la création de nouvelles formes de vie. On est constamment confronté de façon directe à ces questions dans les contextes de sociétés transitionnelles. Il ne s'agit pas uniquement de restaurer la démocratie, mais de dépasser des divisions internes ou des discriminations à grande échelle manquées par les capacités pourtant instituées, tout en sauvegardant la puissance de cet inexpérimentable.

Dans ces situations où l'expérimentation doit prendre un tour non standard, deux exigences pragmatistes se retrouvent néanmoins, mais sur un mode différent. D'un côté, la création d'un milieu tiers susceptible de rendre possibles des apprentissages. C'est notamment la fonction principale de la sociologie des absences qui rend possible un autre type de rapport à l'attention, laquelle, en étant déstabilisée par le hors champ ou l'insu, se laisse affecter par l'indisponibilité du monde. D'un autre côté, la théorisation nécessaire de préconditions pour que les agents concernés puissent tirer parti de ce milieu tiers. C'est l'enjeu notamment des règles d'une contre-pédagogie sociale visant à resensibiliser ou à déjouer la jouissance prédatrice avec ses effets réificateurs. En même temps, ces approches innovantes continuent de s'appuyer sur la persistance d'un socle expérimentaliste jusque dans le savoir du non savoir, à travers l'idée consensuelle d'un inexpérimentable à sauvegarder comme trou dans l'espace de vérité. Cette confiance radicale de l'expérimentalisme non standard dans la subversion du modèle standard est en fait corrélative du renforcement du développement de l'expérimentalisme standard comme source de résolution des problèmes collectifs. Le principe d'expérimentation est poussé au-delà de sa limite, en perdant de vue certaines de ses limites majeures: il suppose la capacité de s'entendre sur des mesures communes, de comprendre la distribution des niveaux de pouvoir et leurs rôles, de prendre du temps pour former des alliances et des significations partagées, enfin de tirer parti d'une faible centralisation ${ }^{54}$. Un dernier front doit donc encore être envisagé actuellement face aux différentes formes d'expérimentalisme analysées, celui qui considère comme un risque inutile la mise en jeu de son capital politique pour privilégier de telles capacités et s'efforce au contraire de mettre un terme au " consensus expérimentaliste ». 


\section{Les sorties du consensus expérimentaliste}

30 On peut en effet pointer actuellement deux types de sorties du «consensus expérimentaliste", lesquelles s'efforcent donc d'offrir des options au consensus expérimentaliste suivant lequel, en cherchant ensemble des solutions, on maximise les chances de capture des solutions innovantes et on crée en même temps les conditions d'une meilleure réception de celles-ci grâce à la procédure collaborative mise en place. De fait, la faiblesse de ce consensus est qu'il s'appuie sur deux présuppositions. D'une part, il suppose - au-delà des capacités qu'il estime toujours déjà données pour réaliser de telles pratiques collaboratives et innovantes - un accord sur la manière de produire ensemble une vérité commune à travers des significations partagées et de nouvelles heuristiques. D'autre part, il s'appuie sur une volonté d'appliquer de manière réflexive ces propositions en allant au-delà des garanties attendues par les identités d'action mises en jeu dans les processus de transformation.

31 Ces deux présuppositions sont en fait indicatives de deux formes de sortie du consensus expérimentaliste. La première consiste à modifier les conditions de production des vérités communes en conférant l'autorité d'action à un pôle expérimentateur autonome, habilité à forcer les cadres habituels et à élargir les possibilités admises dans une trajectoire déterminée. L'autorité est alors perçue comme concentrant une plus grande force d'exploration par son pouvoir de redéfinition des cadres négociés et constamment actualisés par les compromis successifs. La discontinuité ou la bifurcation, l'accélération aussi, dépend d'une autorité indépendante, capable d'impulser une orientation qu'il est ensuite possible de revoir et d'éliminer sur la base de nouvelles données, que la seule négociation ne permettait pas de fournir. Cette option autoritaire n'est pas l'apanage de mécanismes autoritaristes. Elle apparaît tout autant dans les régimes d'urgence où s'exerce une concentration de la décision, que dans les moments d'impasse de la négociation où l'exécutif peut reprendre la main et imposer sa feuille de route.

Une deuxième forme de sortie du consensus expérimentaliste réside dans la rupture de l'approche multi-niveaux où le local est constamment stimulé par le global et réciproquement. Plutôt que de forcer une solution par en haut, cette forme de sortie préconise au contraire une solution par en bas, tout aussi provisoire que la sortie par en haut, mais de nouveau considérée comme potentiellement plus efficace. Dans ce cas, ce n'est pas l'exploration coordonnée de nouvelles options qui prévaut, mais l'exploration isolée de solutions partielles, mais suffisamment appropriables dans un contexte que pour créer un effet d'entrainement sur le plan local. La dimension spatiale est ici primordiale et la communalité de la solution proposée se définit d'abord par le territoire. Le lieu est le laboratoire privilégié par la proximité qu'il crée, l'intensité et la fréquence des échanges, à faible coût de transaction. Cette stratégie peut être couplée avec des processus radicaux de résistance et de déconnexion pour marquer l'autonomie potentielle du lieu, mais elle apparaît aussi sous d'autres modalités, dès qu'est admis le potentiel d'un milieu de concentration (certains diront d'incubation) et de proximité, accompagné d'une forme plus ou moins forte d'auto-gouvernement.

De part et d'autre, ce qui se manifeste dans ces tendances est en fait la recherche d'une unité d'action, là où le consensus expérimentaliste reste sur une représentation faible d'une forme spontanément agrégative d'intérêts potentiellement divergents, mais séduits par le dispositif collaboratif et les avantages qu'il promet. En fait, l'unité 
d'action que présuppose l'expérimentalisme sans la construire réellement, mais en supposant données les capacités de coopération, est ce qui justifie des formes de sorties plus radicales, capables cette fois de donner une réponse forte à l'unité d'action attendue pour mener des changements significatifs. On procède soit par «suripséisation » dans une figure d'autorité de délégation (fixée dans le temps: l'urgence, l'exception, les pouvoirs spéciaux, les décrets), soit par désafiliation au profit d'un souci communautaire ou d'un bien-être partagé (fixé dans le lieu: le monde vécu, les communs).

Un chef ou un peuple comme unité d'action! Ou, encore, la résonnance acceptée d'une volonté générale réinventée ou la divergence volontaire d'une singularité assumée. Ce qu'indiquent ces sorties de l'expérimentalisme, c'est qu'une forme de gouvernance réflexive des systèmes démocratiques s'est mise en place en présupposant la solution au vide de son unité d'action, sur un mode conséquentialiste. Celle-ci n'étant pas préexistante, elle est toujours en cours d'autoréalisation et l'identité politique ne cesse de se redéfinir par la participation à ces mécanismes de recherche commune de solutions. Mais le fait d'être capables de s'entendre ne signifie en aucun cas le fait de pouvoir vivre en commun avec les différences irréductibles qui nous constituent provisoirement et ainsi d'adhérer à une certaine unité citoyenne d'un commun à préserver plutôt qu'à mettre en péril, voire à détruire. On peut certes tenter de concevoir formellement cette unité comme résultant nécessairement d'un processus agonistique qui l'enrichit constamment, mais il faut aussi que la mise en action de cette agonistique puisse non seulement garantir ses effets, mais aussi se travailler comme agonistique sociale, donc se déterminer et se limiter, s'élargir ou se restreindre, donc se gouverner, et pas simplement se manifester sur le mode naturaliste de réactions ou de troubles, qu'on gère en fonction de leur apparition. En suivant cette logique, l'absence de troubles n'appelle aucune action, alors que la perspective d'une agonistique réfléchie devrait susciter une autre forme d'interaction et se préoccuper tout autant du signal que représente l'absence de trouble que du signal fourni par leur intensification. L'atonie sociale, voire les formes avancées de dépression collective, le mal-être au travail par exemple, sont des signaux d'impasses créatives peut-être plus forts que les troubles explicites secouant l'inconfort collectif.

À ces questions, l'expérimentalisme ne peut répondre, dans la mesure où il ne se conçoit pas en fonction d'une action sur les limites de ses modes de gouvernance, mais plutôt comme une action sur les modes de gouvernance démocratiques qu'il cherche à renforcer en stimulant leur agrégation. Il n'a pas de solution, dans la mesure où son modèle considère comme déjà résolues ces questions par un simple effet collectif d'agrégation procédurale : l'émulation produite par l'interaction " procéduralisée » des perspectives de co-élaboration, de comparaison et de co-design suffit à engendrer une unité plurielle, jamais une en soi mais toujours en formation en fonction de sa tâche de détection et de réajustement de ses décisions. Le consensus de la bonne conscience expérimentaliste, y compris avec l'identification platonique de désaccords fondamentaux, fige en fait l'absence d'unité d'action et se projette comme l'ordre communicationnel vers l'idéal d'une communauté de communication sans contrainte où l'on peut a priori toujours finir par s'entendre.

Le risque actuel réside donc aussi dans l'impuissance du consensus expérimentaliste à réagir devant le besoin de remplir ce vide de l'unité d'action, soit par mot d'ordre, soit par démarcation, deux substituts possibles contre la garantie supposée de la 
participation responsable à un intérêt général co-construit. En fait, la véritable leçon de l'inexpérimentable et de la sociologie des absences, est que la limite majeure de l'expérimentalisme réside dans le rapport de croyance qu'il institue comme ordre politique à l'égard de ses capacités, ainsi que dans l'aveuglement corrélatif qu'il entretient à l'égard de sa destruction possible comme norme du pouvoir-faire démocratique. Il ne parvient pas à traverser le vide imaginaire de l'unité d'action qu'il suppose en reconnaissant, d'une part, l'équivoque des capacités qu'aucune règle interne ne peut prémunir contre la répétition des exclusions et des discriminations et, d'autre part, l'idéalisation de cette normativité supposée qui le rend aveugle à sa négation externe. La question la plus urgente pourrait ainsi être celle de savoir si et à quelles conditions il y a une sortie démocratique du consensus expérimentaliste sous ces formes standards et si, précisément, celle-ci ne passe pas par un autre rapport collectif à la supposée disponibilité du savoir-expérimenter. Pas de pouvoir-faire démocratique sans une action liminaire essentielle sur les conditions de constructions de ce pouvoir, une action qui se confronte à l'indisponibilité du savoir-expérimenter, tant à travers l'équivoque des capacités, qu'à travers l'idéalisation de la normativité expérimentaliste.

\section{NOTES}

1. Daniel Cefaï et Danny Trom (dir.), Les formes de l'action collective, Paris, éditions de l'EHESS, 2001 ; D. Cefaï, Pourquoi se mobilise-t-on? Les théories de l'action collective, Paris, La Découverte, 2007.

2. Michael C. Dorf \& Charles F. Sabel, «A Constitution of Democratic Experimentalism », in Columbia Law Review, vol. 98, 1998, p. 267-473.

3. Paul Aligica \& Vlad Tarko, "Polycentricity: From Polanyi to Ostrom, and Beyond", in Governance: An International Journal of Policy, Administration, and Institutions, vol. 25, $\mathrm{n}^{\circ} 2,2012$, pp. 237-262, pp. 245-246.

4. Fritz Scharpf (ed.), Games in Hierarchies and Networks: Analytical and Empirical Approaches to the Study of Governance Institutions, Frankfurt am Main/ Boulder (CO), Campus/Westview, 1993

5. Oliver E. Williamson, The Mechanisms of Governance, Oxford, Oxford University Press, 1996; ainsi que Douglass North, Institutions, Institutional Change and Economic Performance, Cambridge, Cambridge University Press, 1990.

6. Marten Hajer \& Hendrik Hagenaar (eds), Deliberative Policy Analysis: Understanding Governance in the Network Society, Cambridge, Cambridge UP, 2003.

7. C. Sabel \& William H. Simon, «Democratic Experimentalism», in Justin Desautels-Stein \& Christopher Tomlins (eds), Searching for Contemporary Legal Thought, Cambridge, Cambridge University Press, 2017; repris in Columbia Public Law Research Paper, $n^{\circ} 14-549$ (2017).

8. C. F. Sabel, "Dewey, Democracy, and Democratic Experimentalism ", in Contemporary Pragmatism, vol. 9, n², 2012, pp. 35-55.

9. C. F. Sabel \& Jonathan Zeitlin, « Experimentalist Governance », in GR:EEN Working Paper, n 9 , 2012, https://cris.unu.edu/experimentalist-governance, p. 4 (repris dans David Levi-Faur (ed), The Oxford Handbook of Governance, Oxford, 2012).

10. James Bohman, «Democratic Experimentalism », in Social Philosophy Today, vol. 29, 2013, pp. 7-20. 
11. La même règle d'action appliquée de manière récurrente par des unités plus petites modifie progressivement le problème général d'application et permet d'en trouver une solution.

12. C. F. Sabel, « Dewey, Democracy, and Democratic Experimentalism », op. cit., pp. 37 et 41.

13. Ibid., p. 39.

14. Elisabeth Anderson, «The Epistemology of Democracy », in Episteme, vol. 3, n 1-2, 2006, p. 14

15. "Democratic norms of free discourse, dissent, feedback, and accountability function to ensure collective, experimentally-based learning from the diverse experiences of different knowers » (ibid., p. 8).

16. C. F.Sabel, « Dewey, Democracy, and Democratic Experimentalism », op. cit., p. 40.

17. Ibid.

18. Ibid., p. 41.

19. Joëlle Zask, «Le public chez Dewey: une union sociale plurielle», in Tracés, vol. 15, 2008, pp. 169-189, http://journals.openedition.org/traces/753.

20. Ibid., p. 181.

21. Ibid., pp. 180-181.

22. Sur ce point, on précisera la lecture de Henry Georges avec l'article de Christopher England, «John Dewey and Henry George: The Socialization of Land as a Prerequisite for a Democratic Public », in American Journal of Economics and Sociology, vol. 77, n 1, 2018, pp. 169-200 .

23. J. Zask, « Le public chez Dewey : une union sociale plurielle », op. cit., p. 181.

24. H. Wagenaar, Meaning in Action: Interpretation and Dialogue in Policy Analysis, London, Routledge, 2015.

25. Judith E. Innes \& David E. Booher, Planning with Complexity, An Introduction to Collaborative Rationality for Public Policy, London, Routledge, 2010.

26. H. Wagenaar \& Noam Cock, «Understandind policy practices: action, dialectic and deliberation in policy analysis ", in M. Hajer, H. Wagenaar (eds), Deliberative Policy Analysis, op. cit., pp. 139-171, pp. 167-168.

27. J. E. Innes \& D. E. Booher, « Collaborative policymaking: governance through dialogue », in M. Hajer, H. Wagenaar (eds), Deliberative Policy Analysis, op. cit., pp. 33-59, pp. 38-39.

28. J. E. Innes \& D. E. Booher, Planning with Complexity, op. cit., p. 118.

29. Ibid., p. 116.

30. Ibid., p. 35. A theory of collaborative rationality: diversity, interdependence, authentic dialogue (DIAD).

31. Hartmut Rosa, Résonance, Une sociologie de la relation au monde, Paris, La Découverte, 2018, p. 279.

32. Ibid., pp. 281-282.

33. Ibid., p. 278. Une idée présente non seulement chez Herder, Schiller et Humboldt, comme le rappelle Rosa, mais aussi plus radicalement encore chez Alois Riegl, ensuite repris par Lamprecht et Bloch. Ainsi du terme diapason (ibid., p. 280).

34. Ibid., p. 279.

35. J. Zask, « Le public chez Dewey : une union sociale plurielle», op. cit., p. 181.

36. Ibid.

37. H. Rosa, Rendre le monde indisponible, Paris, La Découverte, 2020, pp. 88-89.

38. Ibid., pp. 128-129

39. J. Bohman, « Democratic Experimentalism », op. cit., pp. 7-20.

40. W. H. Simon, "Justice and Accountability: Activist Judging in the Light of Democratic Constitutionalism and Democratic Experimentalism », in Law, Culture and the Humanities, vol. 15, $\mathrm{n}^{\circ}$ 3, 2019, pp. 602-621.

41. Jules Coleman, The Practice of Principle: In Defence of a Pragmatist Approach to Legal Theory, Oxford, Oxford UP, 2001.

42. Robert E. Goodin, Reflective Democracy, Oxford, Oxford UP, 2003 
43. En particulier, on verra les précisions apportées sur ce point par l'article de Shane Ralston, " Dewey and Goodin on the Value of Monological Deliberation ", in Etica \& Politica, vol. XII, 2010, $\mathrm{n}^{\circ} 1$, pp. 235-255.

44. Catherine Iorns Magallanes, «Nature as an Ancestor : Two Examples of Legal Personality for Nature in New Zealand », in VertigO. la revue électronique en sciences de l'environnement, Hors-série $\mathrm{n}^{\circ}$ 22, http://journals.openedition.org/vertigo/16199.

45. Roberto Mangabeira Unger, What Should Legal Analysis Become?, London/New York, Verso, 1996, p. 6.

46. Et pas uniquement leur «vulnérabilité ».

47. R. M. Unger, What Should Legal Analysis Become?, op.cit., p. 165; ainsi que The Self Awakened, Pragmatism Unbound, London/Cabridge, Harvard UP, 2007, pp. 184-185.

48. Boaventura de Sousa Santos \& César Rodríguez-Garavito, «Law, Politics, and the Subaltern in Counter-Hegemonic Globalization », in B. de Sousa Santos \& C. A. Rodríguez-Garavito, (eds), Law and Globalization from Below, Towards a Cosmopolitan Legality, Cambridge, Cambridge UP, 2005, p. 9.

49. B. de Sousa Santos, «Épistémologies du Sud», in Études rurales, vol.187, 2011, pp. 21-50, pp. 34-35; ainsi que Épistémologies du Sud, Mouvements citoyens et polémiques sur la science, Paris, DDB, 2016, pp. 251-252.

50. B. de Sousa Santos, Épistémologies du Sud, op. cit., p. 255.

51. Arturo Escobar, Sentir-penser avec la Terre, Paris, Seuil, 2018.

52. Rita Laura Segato, Contra-pedagogías de la crueldad, Buenos Aires, Prometeo Libros, 2018.

53. Walter Mignolo, The Darker Side of Western Modernity, Global Futures, Decolonial Options, Durham, Duke UP, 2011.

54. David A. Super, «Laboratories of Destitution: Democratic Experimentalism and the Failure of Antipoverty Law ", in University of Pennsylvania Law Review, vol. 157, 2008, n² 2, pp. 541-616.

\section{RÉSUMÉS}

Si l'expérimentalisme démocratique est particulièrement exemplatif du tournant pragmatique des sciences sociales en théorie de la gouvernance, il va bien au-delà du réformisme institutionnel défendu par Sabel, Dorf ou Simon pour se décliner aussi dans des approches plus radicales comme celles de Roberto Unger ou de Souza Santos. La question qui se pose en reconstruisant le spectre de ces propositions est de savoir si elles ont suffisamment anticipé leur propre dépassement pas des formules qui prônent explicitement l'anti-expérimentalisme comme condition de restauration de l'autorité sociale.

\section{INDEX}

Mots-clés : expérimentalisme démocratique, pragmatisme, Sabel, Dewey, Rosa, Unger Index chronologique : Europe 


\section{AUTEUR}

\section{MARC MAESSCHALCK}

Marc Maesschalck est professeur agrégé de philosophie à l'Université catholique de Louvain où il dirige le Centre de philosophie du droit (CPDR) fondé par Jacques Lenoble et André Berten. Il a enseigné en Haïti, au Québec, au Luxembourg, en Suisse et en France. Spécialiste de Fichte et de Schelling, il a publié de nombreuses études en philosophie sociale et politique ainsi qu'en éthique et en théorie du droit. Ses derniers ouvrages sont Reflexive Governance for Research and Innovative Knowledge, (Wiley-ISTE, 2017), La cause du sujet (Lang, 2015), Democracy, Law and Governance (Ashgate, 2010, avec J. Lenoble) et Transformations de l'éthique (Lang, 2010). Ses travaux actuels visent à cerner les conséquences d'une sortie de l'épistémologie eurocentrée sur la théorisation des mécanismes de gouvernance et les relations entre sociétés subalternes et sociétés dominantes. 